\title{
A Conceptual Design and Numerical Analysis for a Small-Scale and Low-Cost Plastic Recycling Machine
}

\author{
Anh T. M. Le ${ }^{1}$, Hoang D. Doan ${ }^{1}$, Loc P. Ngo ${ }^{1}$, Ly T. Huynh ${ }^{1}$, Tuan N. Huynh ${ }^{1}$, Huy T. Phan ${ }^{1}$, and Thanh T. Tran ${ }^{2}$ \\ ${ }^{1}$ Graduate Student, Department of the Global Production Engineering and Management, Vietnamese-German University, 2 Le Lai, Thu \\ Dau Mot City, Binh Duong, Vietnam \\ ${ }^{2}$ Lecturer, Department of the Global Production Engineering and Management, Vietnamese-German University, 2 Le Lai, Thu Dau Mot \\ City, Binh Duong, Vietnam
}

\begin{abstract}
A new conceptual design for a small-scale and low-cost plastic recycling machine is generated by combining melting part and compression process. Starting with one of the outstanding requirements is in terms of an affordable-priced machine that can perform two processes with high accuracy and capacity, some issues related to balancing among quality, capacity and cost of machine occurred during a discussion. After implementing various designing methods such as Quality Function Deployment, Reverse Engineering, Morphological Matrix and Pugh Method, an idea of final concept about using an electric oven and hydraulic system to melt down and compress plastic tile which has a dimension of 300x300x9 mm was created. The design of concept is divided into two parts which are mechanical and electrical systems. In a mechanical section, the technical drawing and simulation are made to see how machine performs under operation. Besides, we examined the forces that applied in the moulds to evaluate the strength of the system. In heating and electricity section, we chose electrical components, designed oven parameters and conducted the heating simulation on the mould. In addition, the heating and cooling time was calculated based on the principles of thermodynamics and heat transfer. Furthermore, the manufacturing plan is created to estimate the essential resources producing a certain number of heat-forming machines. In general, the machine needs to be prototyped for controlling its main function and finding practical issues. After that, some improvements could be made to enhance efficiency and increase capacity by designing an optimal mould to more heat absorb and reduce post process, calculate and design more efficient oven, create faster lock mechanism and other improvements for an automatizing machine.
\end{abstract}

\section{Introduction}

Increasement of plastic waste has been raised an urgent problem to the whole society. The reduction of plastic and invention of machines must be implemented to ease the problem causing by plastic waste [1]. The amount of plastic and their variation need to be handled by developing plastic recycling system. Large-scale recycling plastic plants are built in developed countries because a huge amount of plastic waste is collected and classified. While plastic waste is being burned and buried in many developing/emerging countries. To reduce the proportion of plastic to be incinerated, this conceptual design contributes an improvement for plastic recycling machine in Vietnam. Sorting, cleaning, drying, shredding, heating and forming are main steps in the plastic recycling process. Climate change and plastic pollution have impacted governments' awareness of countries. There are many social media campaigns were conducted to increase citizens' awareness about household sorting of waste at source. However, these campaigns were implemented asynchronously and almost citizens did not recognize benefits of classifying garbage. The change in habit has happened slowly and plastic recycling rate was low or no data. This is the reason why investment in plastic recycling plants is quite risky. The cost of recycling plastic machines is range from $\$ 20000$ to $\$ 100000$ depending on the capacity and resin type [2]. Enterprises also pay for workforce, machine maintenance, and energy consumption. Profit problems make it hard to attract investment from large-scale enterprises.

Recycling plastics to make base products is the potential direction which is getting attention from small-scale enterprises, start-up company, universities and nongovernment organizations. Recycling plastic tile is a type of base product which has promised future in furniture and construction field. Because of small size, recycling plastic tiles have more advantages to produce in every household. The Precious Plastic project by Hakkens et al [3] suggested a compression machine which is made from recycling components. Although the cost of this machine is low, there are many factors having to be considered. The components are recycled from discarded machines which are very hard to mass production. The productivity is low because the machine just has one simple mould inside. The heat loss is high because of lack of thermal 
isolation layer. The ReForm project from Evergreen Labs et al [4] aims to create a household recycling process which includes shredding, heating, forming, pressing machine. However, this project still cannot complete because of many defects appear in experiments. The shrinkage during the cooling process is significant and lead to deforming during the cooling process and air enclosures. The arrangement of heating elements made a distribution of heating temperature is uneven. The heating temperature is also hard to control and lead to burning plastics. There is a problem related to quantification in production such as maximum dimensions of tile, weight of material inside cavities, colour pattern.

This research aims to create a conceptual design and numerical analysis for machine elements of plastic recycling machine which can solve problems from previous projects. The technical parameters of this machine are converted from a voice of customer (Evergreen Labs) by Quality Function Deployment (QFD) method. The heating elements are rearranged and archived uniform temperature distribution by thermal simulation. The heating time and cooling time of mould set on the principles of thermodynamics and heat transfer. The design of mould is based on dimensions of products and try to solve not only quality problems but also the productivity of the machine. The final design consists of main components of this machine and their blueprints which can be used for mass production directly.

\section{CONCEPTUAL DESIGN}

\subsection{Convert Requirements to Technical Parameters}

Based on Evergreen Labs requirements' list, the Quality Function Deployment tool makes a system engineering process in which product development process is linked to quality of product as defined by Evergreen Labs [5]. From that point, the engineering parameters of products may be posed to satisfy those expectations. Through result of QFD report, readers can pay their attention effectively on key criteria rather than overviewing without priority values. By this way, machine builders can resolve each engineering specification in an orderly manner from highest importance to lowest one. The most three customer demands after QFD consideration include product dimension, output quality (no bent, no air bubbles), and high productivity. To resolve them, five engineering specifications shall be observed, consisting of the output dimension, bending angle, pressing force, air-filled density, and the maximum diameter of airbubbles. All of them are necessarily reduce as much as possible. Turning on the creation of ideas of product design, by using technical specifications derived from QFD, Reverse Engineering method has been applied to detect function process and its needed function parts [6] (shown in Fig. 1). Morphological Matrix which concerns the analysis and permutations of possible solutions of machine elements is used for construction elements development process [7]. There are three options for the machine consisting of an injection-molding machine, injection-machine with rollers, and compression machine with multi-mold. Nevertheless, Pugh method eliminates two options with lower rating than compression machine with multi-mold.

For further evaluation, Computer Aided Design/ Computer Aided Engineering (CAD/CAE) application and drawing built help to demonstrate Pugh's method result of compression molding machine with multi-mold

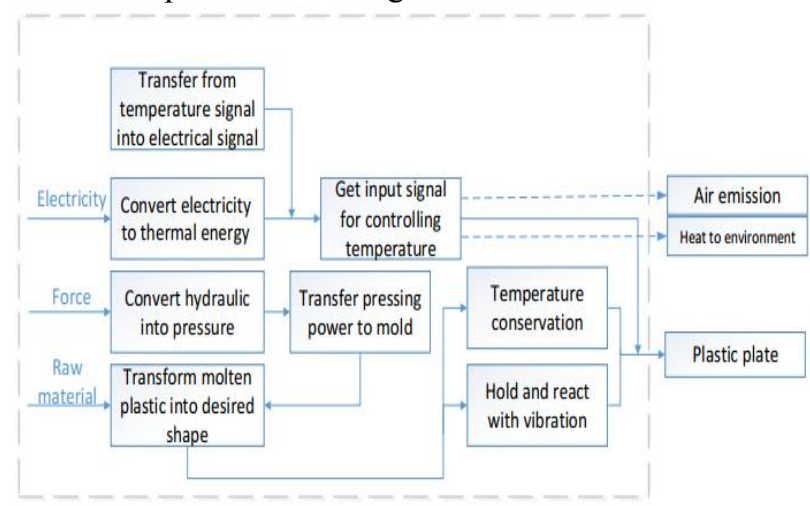

Fig. 1 Functional process of Healing-forming plastic machine

\subsection{Heating technology}

The heating process in the oven up to now, there have been many different technologies which can provide a very high efficiency and short heating time such as microwave, heating element, inductor and infrared. However, every technology has specific disadvantages which affect the heating process of recycling plastic. The microwave cannot be able to melt down plastic because it can be used to heat up the material which contains the water molecule inside only [8]. For the inductor oven, it requires a kind of mold material called ferromagnetic that causes more difficulties in the mold manufacturing [9]. The infrared oven is not suitable as well because of high cost and power consumption in the current scale. Therefore, the electric oven using heating element is the most enough and feasible choice for heating technology in this research.

\subsection{Mould Design}

Conceptual design and optimizing design

To approach the desired capacity with the compression forming method, two design concepts have been evaluated. In the first concept, plastic is formed inside a rectangular box by using a single mold whose dimension is $300 \times 300 \times 50 \mathrm{~mm}$. The main advantage of this concept is the height of the product is flexible and can be changed easily by changing the dimension of the fixture used in slicing process. However, this concept requires an additional slicing machine and a fixture for slicing process, which could increase the cost for equipment and the final weight of the whole system.

For the second concept, a set of molds is used to make separated tiles with the dimension of $300 \times 300 \times 9 \mathrm{~mm}$. 
This concept has disadvantages such as lack of ability to change dimensions of product and the additional cost for manufacturing five molds. However, additional slicing machine is not required. Moreover, cycle time and quality of the final product are controlled. With the good result from the practical experiments of Evergreen Labs with the similar mold dimension for only one mold, the second concept is chosen. In this study, the method of injection molding in which the plastic material will be melted down inside the barrel and then it is injected into the closed mold for forming the final product is used [10]. Because of the thin thickness of the final product, the main force, in this case is to $30,000 \mathrm{~N}$ which is equal to the force used in experiments of Evergreen Labs. By using Solidworks Modeling software, a concept for a set of molds has been designed as Fig. 2a in which five layers of mold will be compressed together at the same time. The dimension of the product is determinate by the shape of cavities inside the mold and the bottom face of the upper mold pressing on lower mold. One of the most critical points of this concept is the guiding system for six layers have to be pressed at the correct position to avoid the exceeding material and create the desired shape of the product. There are four guide pins are located at the four corners of the base mold for guiding the whole system. Additionally, the guide pins are used as the fixture which keeps the springs inside the set of molds. Springs are used to create the forces to separate each layer of molds after releasing the clamp. There are three kinds of mold in each set of molds (as in Fig. 2b) which are layer mold (LM), base mold (BM) and top cover plate (TC). BM and LM have the same inner dimension for forming the shape of the product. Because the whole heat-forming system will be operated by local civilians and the amount of input plastics is controlled by its weight, the exceeding material cannot avoid. Therefore, the solution is to reduce the length of each bottom surfaces of LM and TC to $298 \mathrm{~mm}$ for keeping these materials. Theoretically, BM and TC are developed based on the design of LM (as Fig. 3a) with some major modifications. The ears of BM thickness have been increased $10 \mathrm{~mm}$ for carrying the guide pins and the rest of molds have ears' thickness is 5 $\mathrm{mm}$. Consequently, the thickness of the BM is also increased leading to the weight of BM is increased. To solve this problem, four slots have been made in the bottom surface of the BM for reducing weight. (a)

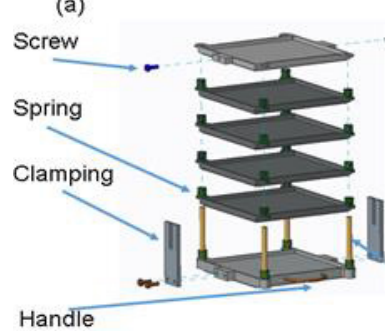

(b)

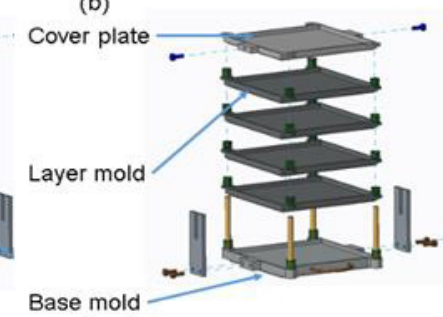

Fig. 2. (a) Main components;

(b) three kinds of mold in set of molds

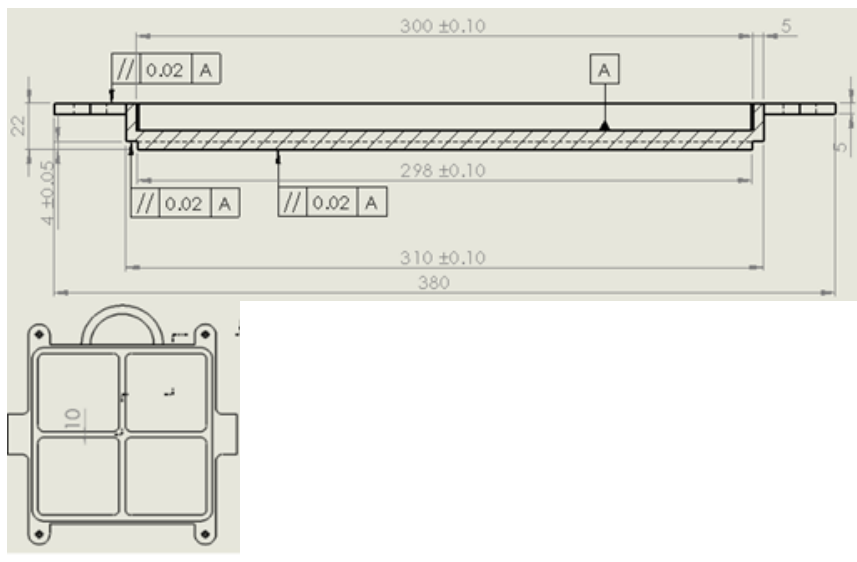

Fig. 3. (a) Detail design of layer mold ; (b) Shape of base mold

Another modification of BM is two thick ears in two sides of the mold for the location the clamping system. The clamping system will be fixed at the BM and freely slide to the holding ears of TC. The clamping of TC will be fixed after the set of molds is fully compressed. Beside of two additional ears for clamping, TC has been increased the thickness of the bottom layer to $12 \mathrm{~mm}$ because of the pushing head has the smaller touching surface which lead to the increase of pressing force to the $\mathrm{TC}$ and may lead to the fracture of TC.

- Nominating material and structure analyses

As mentioned, this set of molds has the same working method as injection molding machine where the pressing force is applied to press and close the mold, and the result is an impact between each surface of the mold. This is the reason why medium carbon steel is not used for making injection molds. However, in this machine, the compressing force is much lower than the compressing force of normal injection machine and medium carbon steel is a decent option. For reducing cost and widely approach in local markets, AISI 1045 steel, which contains $0.43 \%$ to $0.5 \%$ Carbon, is referred [11]. Moreover, the hardness of AISI 1045 steel can be increased by surface heat treatment to reach the hardness approximate 55HRC [12] and the Yield Strength of AISI 1045 steel is $2.42 \mathrm{e}+08 \mathrm{MPa}$. To confirm the suitability of AISI 1045 steel in the concept design, Solidworks Simulation is used for checking the internal stress of the mold and the deformation of molds while being compressing. Regarding the design of LM, the TC is the most critical mold in which the force of springs below focusses on the ears of LM.

Fig. 4 describes the force distribution on the TC mold in which the red arrows representative for the pressing force from TC and equal to the compressing forces $(30,000 \mathrm{~N})$. Blue arrows representative for the counter force of the plastic of below mold to the bottom surface of top cover mold. Due to lacking practical experiment, this force is assumed to equal to 15,000 (50\% of compressing force) as green area represents the touching surface of this mold to the edge of next mold. 


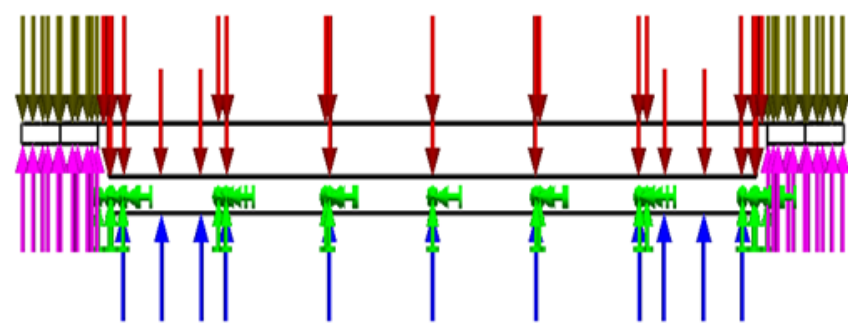

Fig. 4. Force distribution of top cover mild

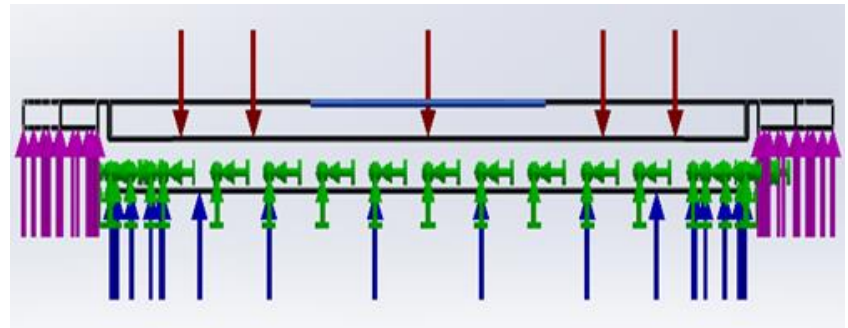

Fig. 5. Force distribution of top cover mold

and it is fixed. Brown and purple arrows represent for the force caused by the springs. By using the medium spring from the Mitsumi with the length of $25 \mathrm{~mm}$ without compression and $12.5 \mathrm{~mm}$ when it is fully compressed, each spring generates nearly $390 \mathrm{~N}$. Therefore, the magnitude of brown arrows and purple arrows are $390 \mathrm{~N}$ and $1,560 \mathrm{~N}$ respectively. The analysis of $\mathrm{BM}$ is ignored because BM is similar with LM and the bottom surface of BM is even thicker than LM. For analysing TC, the same counterforce is applied. However, the pressing area is narrowed down to the circle (which is the profile of pressing head) with the diameter is $240 \mathrm{~mm}$. The upper spring force is removed, and the bottom spring force is increased to $1,950 \mathrm{~N}$ caused by 5 springs linear connection (as Fig. 5).

\subsection{Heating Time Calculation}

There are the parameters for calculating the heating time of heat-forming machine: $\mathrm{m}$ is the mass of materials $(\mathrm{kg})$; $\mathrm{C}$ is heat capacity of materials $(\mathrm{J} / \mathrm{kgK})$; $\mathrm{T}$ is temperature $(\mathrm{K})$; Q is thermal energy transfer $(\mathrm{J})$; $\mathrm{t}$ is time $(\mathrm{s}) ; \rho$ is density of material $(\mathrm{kg} / \mathrm{m} 3)$; $\mathrm{V}$ is volume of material (m3); $\mathrm{P}$ is pressure (atm); $\mathrm{n}$ is mol number (dm3). The dimension of:

The oven is $500 \times 500 \times 500 \mathrm{~mm}$

A plastic plate is $300 \times 300 \times 9 \mathrm{~mm}$

A layer mold is $300 \times 300 \times 50 \mathrm{~mm}$

The volume of pressing plate is $1.8685359 \times 106 \mathrm{~mm} 3$

The total volume of chamber and guide plate is $1.8005518 \times 107 \mathrm{~mm} 3$

The volume of base mold is $1.0834136 \times 106 \mathrm{~mm} 3$

Qplastic $=$ mplastic $\times$ Cplastic $\mathrm{x} \Delta \mathrm{t}=840000(\mathrm{~J}$

Qmold $=$ mmold $\times$ Ccarbon-steel $x \Delta t=3323481(J)$

$$
\text { Qair }=\text { mair } \times \text { Cair } \times \Delta t=17574(\mathrm{~J})
$$

Sum of thermal energy transfer

$$
\mathrm{Q}=\text { Qplastic }+ \text { Qmold }+ \text { Qair }=4181 \times 103(\mathrm{~J})
$$

Thermal energy supply is calculated based on Joule Lenz law:

$$
\mathrm{Qs}=\mathrm{Pt}=3600 \mathrm{t}
$$

In this case, we assume the efficiency of energy conversion is $100 \%$.

Thermal equilibrium equation:

$$
\mathrm{Q}=\mathrm{Qs}=4181 \times 103=3600 \mathrm{t}
$$

Then the heating time $t$ is 1161 (s). In other words, the needed time for heating plastic from $30 \mathrm{oC}$ to $180 \mathrm{oC}$ is 19.34 minutes.

\section{Numerical Simulation and Analysis}

\subsection{Heating Element Arrangement}

- Oven troubleshooting

The heating element in the electric oven is normally arranged at the top and bottom of the oven where the heat is distributed unevenly.

As Fig. 6a, the middle layer of plastic (layer 3) has not absorbed enough heat. It leads to the middle layers cannot be molten down and a failure result in consequently. For all that reason, the simple act of rotating the oven an angle of 90 degrees to change the position of the heating element and also the thermal flow. The modification is described in the Fig. $6 \mathrm{~b}$, the position of the heating element makes the heat distribute evenly for the entire mold from both sides. On the other hand, the thin layer of plastic is separated by a mold layer which can conduct enough heat for the heating process because of the high thermal conductivity of metals. To verify the result of this modification, the thermal simulation is performed on the next section to observe the heat flow and the temperature in the mold to ensure the good condition and result of a heating process.

- Thermal simulation

In this section, the thermal simulation is performed on one mold to track down the direction of the thermal flow inside the mold and to examine whether it is transferred enough heat. First, several constraints are put into the model to archive the accurate and expected result (Fig. 7a). The blue line indicates the direction of convection to the environment. Convection is a phenomenon where the air transfers from the high pressure to the low-pressure zone namely from high temperature to low-temperature zone. The red line is the position of the heat load put on the mold. The yellow line is the flow direction of heat transfer from oven to the mold. 


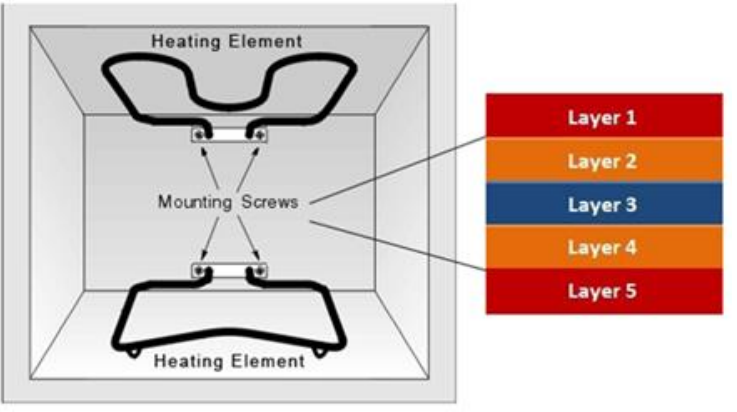

(a)

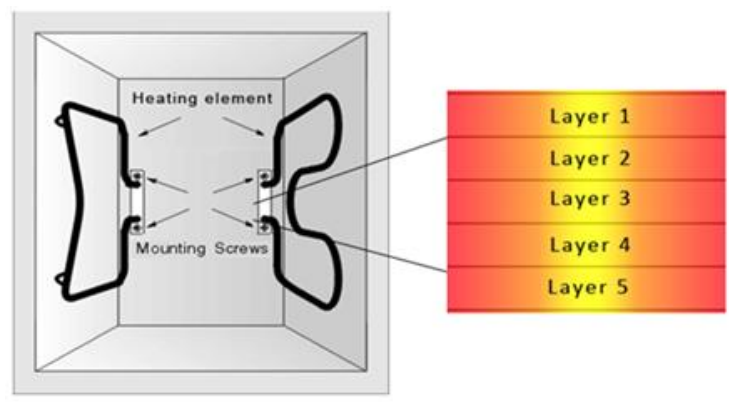

(b)

Fig. 6. (a) Heat is distributed unevenly by top-down arrangement ; (b) Heat is distributed evenly left-right arrangement

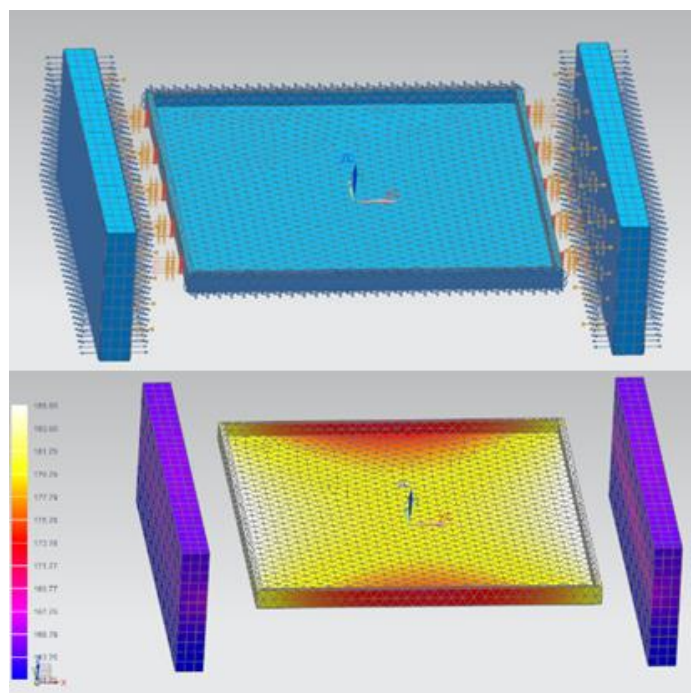

Fig. 7. (a) Thermal contraints ;

(b) thermal distribution in simulation

The thermal constraints and thermal distribution are described in the mold in the Fig. 7b. The hottest zone (white zone) approximately 185 Celsius degrees is the two edges near the oven wall. The coldest zone (red zone) is the rest two edges which has temperature of nearly $175 \mathrm{oC}$. Because the melting point of polyethylene plastics spreads over the range from $120 \mathrm{oC}$ to $180 \mathrm{oC}$, so with $175 \circ \mathrm{oC}$ compared to $180 \mathrm{oC}$, this result is good enough and the model will work sufficiently. In conclusion, the thermal simulation result proves that the modification of heating element position is feasible and can adapt the heating requirement for the good outputs.

\subsection{Mold Simulation}

By using Solidworks simulation, the maximum stress of top cover mold focuses on the edge of the mold where the pressing force has the impact on both bottom surface and side surfaces. The maximum value of internal stress of top cover mold is $1.78 \mathrm{e}+08 \mathrm{MPa}$ and it less than the maximum yield strength of AISI 1045 steel which is $2.42 \mathrm{e}+08 \mathrm{MPa}$. For deformation analysis, Solidworks Simulation has carried out the result as Fig. 8a in which the red zone is the high deformation zone of this mold with the value approximate $0.158 \mathrm{~mm}$ and the deformation increase gradually from edges of the mold into the center of the mold. Therefore, the center of top cover mold is the center of distribution of compressing force. Regarding to the analysis results of TC, the results are quite familiar with results of top cover mold in which the value of internal maximum stress is $1.25 \mathrm{e}+08 \mathrm{MPa}$ and it is slightly smaller than the result of top cover mold and the reason may come from the distribution of the force on TC is only focus on the bottom surface and the increase of bottom thickness to $12 \mathrm{~mm}$. Without the pressure of plastic to the side surface, the shear stress on the edge of the mold is reduced. The same phenomena for deformation result in which the maximum deformation is $0.14 \mathrm{~mm}$ at the centre of TC (as Fig. 8b).

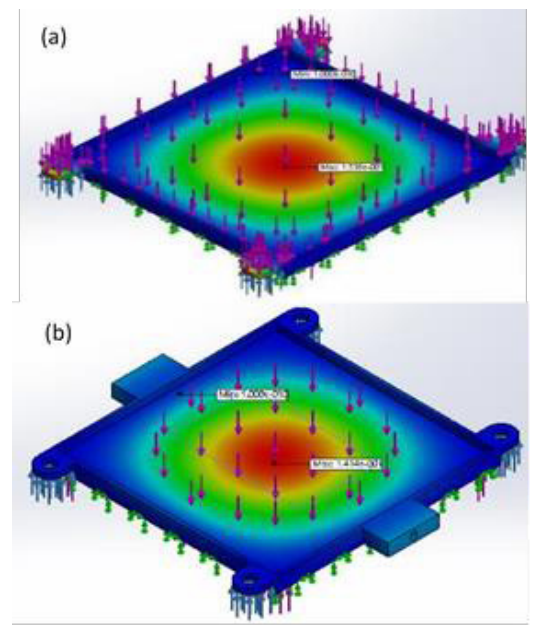

Fig. 8. (a) Top view deformation result of top cover mold; (b) Bottom view deformation result of top cover mold arrangement

\subsection{Final concept design}

The final concept (shown in Fig. 9) is developed from compression technology which is one of the molding technologies that applies force to the preheated material to manufacture metal and plastic object. The raw 
materials for a heating-forming machine in this paper is shredded plastic particles. The main concept of plastic molding is putting a molten plastic into the mold cavity so that shape of plastic can be achieved with the adjustment of calculated temperature and pressure. The mold is then closed, and pressure is applied to force the molten plastic to fill the cavity. The pressure and heat are kept until the plastic is formed into the desired shape. After melting and compressing, set of molds is locked by clamping mechanism and taken out for cooling down. With the set of 5 molds, the cycle time of the machine is reduced because the cooling process would be taken outside of oven instead of putting molds insides. For achieving the realistic design, components are designed for easy manufacturing instead of making hard and unique shapes with local suppliers so that the manufacturing cost could be reduced. In addition, some components which currently exist on the market are also used for designing based on 3D software of Misumi Company.

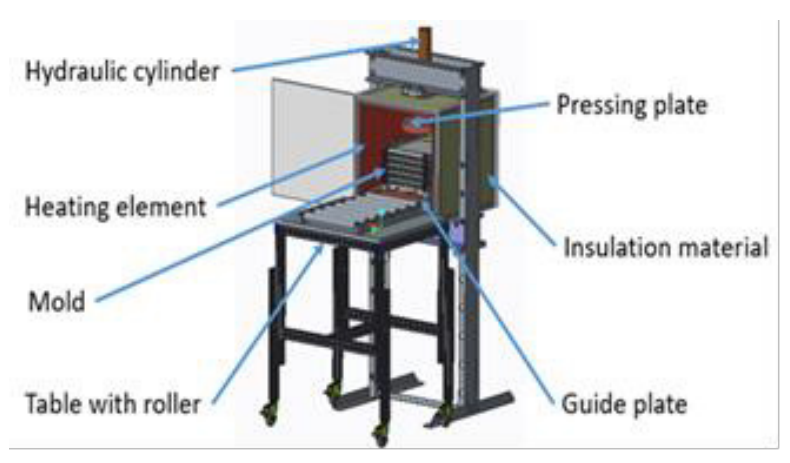

Fig. 9. Final Prototype for the machine

\section{Conclusion and Discussion}

Although this conceptual design is referenced from compression machine of Precious Plastic project, there are many improvements were defined and tested by simulation software. The problems related temperature (uneven melting, burning, Granular patterns) are solved by a new arrangement of heating elements. The heating elements change from top-down arrangement to left-right arrangement and make thermal flow distributed evenly. The hottest zone is approximately $185 \mathrm{oC}$ and the coolest zone is about $175 \circ \mathrm{C}$. This temperature range is suitable to melt polyethylene (PE) and polypropylene (PP) plastic. The length of each bottom surfaces of LM and TC is $298 \mathrm{~mm}$ to avoid excess material. Every mold has 4 ears and connects with 4 pins to guide force of the hydraulic system. The deformation of products in cooling process prevents the clamping which is fixed by tightening bolts. Every set of molds include 5 molds to increase the productivity of machine, the cycle time is 19.34 minutes per product based on the result of heating and cooling time calculation. By simulation force distribution of mold, the center of top cover mold is the center of distribution of compressing force but still in the limit yield strength of
AISI 1045 steel. The set of mold achieves durability to use in a long time. Beside improvements, this research is still in the conceptual phase in which further experiment on the prototype of components should have been conducted. The final dimension of mold, arrangements of the heater is tested by simulation software and some assumptions. The heating time and cooling time are calculated totally based on theories of thermodynamics, then they need to do experiment in laboratories later. The weight of material inside cavities of molds must be measured carefully based on resin type. Generally, the compression machine using multi-mold of five plates can meet Evergreen Labs requirements, especially in terms of output quality, product dimension, productivity, and durability. The final option of a plastic heating-forming machine has already been compared to other heating methods as well as plastic treatment ways; hence, it may pose not only advantages but also disadvantages of solution one-to-one and result in the most proper option for PP and PE plastic recycling. In view of economic, the equipment may be quite proper to a small-scale operation such as households, scrap merchant bases, and even recycling factory. Furthermore, to enhance its performance, buyers can customize and improve it by expanding suitable applications such as adding ventilation to gather fume exhausted generated during processing, two-hand trigger to ensure safety

\section{References}

1. Elo, Kristofer \& Karlsson, Julia \& Lydebrant, Kristian \& Sundin, Erik. (2009). Automation of Plastic Recycling - A case study.

2. Plastic recycling machine price. Retrieved from Alibaba:

https://www.alibaba.com/showroom/plasticrecycling-machine-price.html.

3. Dave Hakkens, (2017). Precious Plastic Project. Precious Plastic Community. Retrieved from Preciousplastic: https://preciousplastic.com/.

4. ReForm Project, (2017). EGL Prototyping. EverGreen Lab. Retrieved from Google: https://sites.google.com/evergreenlabs.org/reform/pr oject-overview.

5. Shahin, Arash. (2018). Quality Function Deployment: A Comprehensive Review.

6. Müller, H.A., Orgun, M.A., Tilley, S.R., Uhl, J.S., (1993). A reverse-engineering approach to subsystem structure identification. J. Softw. Maint: Res. Pract. 5 (4), 181-204.

7. Ostertag, O., Ostertagová, E., \& Huňady, R. (2012). Morphological Matrix Applied within the Design Project of the Manipulator Frame. Procedia Engineering, 48, doi:10.1016/j.proeng.2012.09.544

8. Vollmer, M. (2004). Physics of the microwave oven. Physics Education, 78

9. Sweeney, M., Dols, J., Fortenbery, B., \& Sharp, F. (2014). Induction Cooking Technology Design and 
Assessment. ACEEE Summer Study on Energy Efficiency in Buildings, 9-370.

10. S. Kulkarni, Robust Process Development and Scientific Molding (Theory and Practice), Second edition, Hanser, 2010

11. MATBASE, C45 (EN) / 1.0503 (EN) Mediumcarbon Steel. Retrieved from Matbase: https://www.matbase.com/materialcategories/metals/ferrous-metals/high-gradesteel/material-properties-of-high-grade-steelc45.html\#properties

12. A. Ibrahim, M. Sayuti, Effect of Heat Treatment on Hardness and Microstructures of AISI 1045, Trans Tech Publication, Switzerland, 2015 